\title{
Pentaquark implications for Exotic mesons
}

\author{
T.Burns ${ }^{1}$ and F.E.Close ${ }^{2}$ \\ Peierls Centre for Theoretical Physics, University of Oxford, \\ 1 Keble Rd., Oxford OX1 3NP, UK \\ and J.J.Dudek ${ }^{3}$ \\ Thomas Jefferson National Accelerator Facility, Newport News, VA 23606, \\ USA

\begin{abstract}
If the exotic baryon $\Theta^{+}(1540)$ is a correlated $u d u d \bar{s}$ with $J^{P}=\frac{1}{2}^{+}$, then there should exist an exotic meson, $J^{P}=1^{-} \vartheta^{+}(S=+2) \rightarrow K^{+} K^{0}$ $\sim 1.6 \mathrm{GeV}$ with width $O(10-100) \mathrm{MeV}$. The $\pi_{1}(1400 ; 1600)$ may be broad members of $\mathbf{1 0} \pm \overline{\mathbf{1 0}}$ in such a picture. Vector mesons in the $1.4-1.7 \mathrm{GeV}$ mass range are also compared with this picture.
\end{abstract}

\footnotetext{
1e-mail: burns@thphys.ox.ac.uk

2 e-mail:f.close@physics.ox.ac.uk

3 e-mail:dudek@jlab.org
} 


\section{Introduction}

If the narrow $\Theta^{+}$seen in $n K^{+}$and $p K^{0}$ is confirmed as a pentaquark, then correlations among quarks in Strong QCD play an essential role. There is considerable literature recognising that $u d$ in colour $\overline{3}$ with net spin 0 feel a strong attraction $[1,2]$. We denote this $\left([u d]_{0}^{\overline{3}_{c}}\right)$, the subscript denoting its spin, the superscript being the colour and the ( ) denoting the quasiparticle. Ref. [3] considers the following subcluster for the pentaquark: $\left([u d]_{0}\right)\left([u d]_{0}\right) \bar{s}$ with a $P$-wave between the assumed bosonic $(u d)$ correlations. By contrast ref. [4] assumes that the $\left([u d]_{0}\right)$ seed is attracted in $P$-wave to a strongly-bound "triquark" ([ud $\left.]_{1}^{6_{c}} \bar{s}\right)$. These models assign the $\Theta^{+}$to $\overline{\mathbf{1 0}}$ of flavour.

An essential feature of these dynamics is that in $S$-wave the chromomagnetic repulsion of like flavours destabilises the configuration such that decay to meson + baryon in $S$-wave has such a large width as to be effectively nonexistent [5]. It is in the $P$-wave that potentially interesting pentaquarks emerge.

Mixing between the two configurations $\left([u d]_{0}^{\overline{3}_{c}}\right) \bar{s}$ and $\left([u d]_{1}^{6_{c}} \bar{s}\right)$ has been shown to lead to an eigenstate of low mass, which may be identified with the $\Theta(1540)$ $[6-8]$. Further, this mixing potentially stabilises the $u d \bar{s}$ configuration, underpinning the metstability of the $\Theta[8]$.

The point of departure for this paper is to note that if either of these correlations is realised empirically for the $P$-wave, then on model independent grounds one can replace $\left([u d]_{0}^{\overline{3}_{c}}\right)$ by $\bar{q}$, which implies the existence of $\overline{\mathbf{1 0}}$ and $\mathbf{1 0}$ exotic mesons. A specific example is the analogue of $\Theta^{+} \equiv(u d \bar{s})(u d) \rightarrow \vartheta^{+} \equiv(u d \bar{s}) \bar{s}$. While many of these may be broad and unmeasurable, we shall suggest that if the mixing that lowered and stabilised the $\Theta$ configuration in ref [8] applies, then this leads to an observable $J^{P}=1^{-} \vartheta$ with strangeness $=+2$, together with other $J^{P C}=1^{- \pm}$states with rather characteristic signatures. If the $\Theta^{+}$should survive high statistics data and with a width of $\sim 1 \mathrm{MeV}$, then the observation or otherwise of such mesons may help to discriminate among models for the dynamical origin of that metastability. Recent proposals [8] to explain the anomalously narrow width of the $\Theta^{+}$ought to carry over to the meson world, although due to greater phase space we expect that the meson analogue $\vartheta^{+}$will have width of order $10-100 \mathrm{MeV}$. Analogues with $J^{P}=0^{-}, 2^{-}, 3^{-}$also arise but are expected to be broad and unobservable.

The possibility that the $\pi_{1}(1400)$ and $\pi_{1}(1600)$ [9] could belong to these multiplets is discussed. In addition, the pattern of vector mesons in the $1.4-1.7 \mathrm{GeV}$ mass region [9] may also have some overlap with these ideas.

\section{Exotic Mesons}

The idea that mesons beyond $q \bar{q}$ exist is not new. Jaffe [10] noted that the attractive forces alluded to above lead to a low lying $q q \bar{q} \bar{q} S$-wave nonet. There is good evidence that the scalar mesons below $1 \mathrm{GeV}$ are intimately related to such a picture [11] and the idea of these as correlated diquarks has been resurrected 
by ref [12]. Models of multiquark mesons typically predict a large number of states, in various flavour multiplets and spin states. This usually includes exotics, whose flavour or $J^{P C}$ cannot be constructed from $q \bar{q}$. Any model of exotics must face the fact that there is at most only a handful of such candidates. The full set of flavour representations from $\mathbf{3} \otimes \mathbf{3} \otimes \overline{\mathbf{3}} \otimes \overline{\mathbf{3}}=(\overline{\mathbf{3}}+\mathbf{6}) \otimes(\mathbf{3}+\overline{\mathbf{6}})$ is:

(i) $\overline{\mathbf{3}} \otimes \mathbf{3}=\mathbf{8} \oplus \mathbf{1}$, Jaffe's original nonet

(ii) $(\mathbf{6} \otimes \mathbf{3}) \oplus(\overline{\mathbf{3}} \otimes \overline{\mathbf{6}})=\mathbf{1 0} \oplus \mathbf{8}_{\mathbf{a}} \oplus \overline{\mathbf{1 0}} \oplus \mathbf{8}_{\mathbf{b}}=\mathbf{1 8} \oplus \overline{\mathbf{1 8}}$, the decuplets into which

ref. [13] assign the $\pi_{1}(1400)$ state [9], and accompanying octets, and

(iii) $6 \otimes \overline{6}=\mathbf{2 7}+\mathbf{8}+\mathbf{1}$

All such states were included in the original study of $[q q][\bar{q} \bar{q}]$ in $S$-wave [10]. Within the dynamical assumptions made there, all states other than the nonet (i) were predominantly expected to be very broad and effectively unobservable. With $L=1$ in the system there are many multiplets with negative parity, $J=0,1,2,3$ and $C= \pm$. Either all the states are broad and unobservable or some organising principle is required if one wishes to identify one or two states as members, as e.g. [13], and explain away the remainder. We examine the $q q \bar{q} \bar{q}$ systems in $L=1$ in light of the recent interest in diquark correlations, focussing on the supposed dominance of "good" diquarks over "bad" [5]. We shall see that the correlation of triquark-antiquark (and charge conjugate) leads to a limited spectrum of $J^{P C}$ states, in particular suggesting a lowering of the $1^{- \pm}$multiplets to mass scales akin to those of the $\pi_{1}(1400 ; 1600)$ claimed in $\pi \eta$ and $\pi \eta^{\prime}$ respectively ${ }^{4}[9]$.

\section{The $\vartheta^{+}$: an exotic meson analogue of the $\Theta^{+}$}

If the $\Theta^{+}$is confirmed as a resonant state with $\Gamma \sim 1 \mathrm{MeV}$, then the stability of the correlations, at least in $P$-wave, raises interesting questions for the existence of observable meson analogues in representations $\mathbf{1 8}$ and $\overline{\mathbf{1 8}}$. In the $(u d \bar{Q}) \bar{q}$ correlation, at least, the same dynamics that lead to $\Theta^{+}$in $\overline{\mathbf{1 0}}$ imply a $\overline{\mathbf{1 0}}$ of mesons which will include a $\vartheta^{+}$with strangeness +2 , and its 10 antiparticle with strangeness -2 . In a later section we will show that a dynamical picture in which a triquark-antiquark (and charge conjugate) are in $L=1$ suggests that the $1^{-( \pm)}$multiplet lies lowest.

Neither of the correlations of [3] or [4] alone derives either the low mass or width of the $\Theta^{+}(1540)$ readily [15]. The triquark correlation in ref [4], with the $[u d]$ in the $(u d \bar{s})$ being in the configuration $[u d]_{1}^{6}$ was chosen for its maximal attraction. However, it has been widely noted $[6-8]$ that mixing with the $[u d]_{0}^{\overline{3}}$ via either one-gluon exchange, instanton forces or the effect of virtual $K N$ loops, leads to one eigenstate that is lower in energy than either of the unmixed states. That this could cause a decoupling of the $\Theta^{+} \rightarrow K N$, along the lines suggested in ref [16], is possible but has not been demonstrated (and this mechanism has problems if virtual $K^{*} N$ loops are included).

\footnotetext{
${ }^{4}$ We refer the reader to [14] for an analysis of the experimental data that disputes a resonant interpretation for the $\pi_{1}(1400)$
} 
The energy levels in such a situation are interesting. Ref [4] consider the $u d \bar{Q}$ correlation to be $\left([u d]_{1}^{6} \bar{s}\right)$ for which the chromomagnetic forces are highly attractive, the attractive interaction between $[u d]_{1}^{6}$ diquark and antiquark compensating for the reduced diquark attraction relative to $[u d]_{0}^{\overline{3}}$. The correlation $\left([u d]_{0}^{\overline{3}} \bar{s}\right)$, where there is no such compensating diquark-antiquark hyperfine interaction, is relatively disfavoured. However, the same chromomagnetic forces cause a mixing between these two configurations and the emergence of a light and heavy eigenstate. Ref [8] argues that including both gluon exchange and instanton forces in the mixing analysis, leads to effective masses for the light eigenstate $u d \bar{s} \sim 750 \mathrm{MeV}$ and $m[u d] \sim 450 \mathrm{MeV}$. The reason for this being some $350 \mathrm{MeV}$ more bound than the lightest $u d s \Lambda$ state is that the gluon and instanton forces are twice as attractive in the $q \bar{q}$ channel than in the $q q$ case. Further, as $\mathrm{m}(u d \bar{s}) \leq m(K)+m(d / u)$, for constituent quark mass $m(q)$, the pentaquark cannot dissociate into the $K u(d)$. It is proposed [8] that rearrangement is suppressed in $L=1$ with the result that the $\Theta^{+}$is metastable.

The reduced mass of the $750 \mathrm{MeV}$ triquark and $450 \mathrm{MeV}$ diquark is $280 \mathrm{MeV}$. The similarity of this to the strange quark in the $\phi$, which is $\sim m(\phi) / 4=$ $255 \mathrm{MeV}$, suggests a similar price for $L$-excitation in the two systems. Using $m\left(f_{2}(1525) / f_{1}(1420)\right)-m(\phi) \sim 400-500 \mathrm{MeV}$ as a measure of the orbital excitation energy ${ }^{5}$ giving the mass scale for $\Theta^{+} \sim 1600-1700 \mathrm{MeV}$. Spin orbit splitting might reduce this to $1540 \mathrm{MeV}$ [18].

But now consider the $[u d]$ accompanying the $u d \bar{s}$ in the $P$-wave: we can replace this by any of $\bar{u}, \bar{d}$ or $\bar{s}$ to form a meson. Were we to do so for any combination $q_{i} q_{j} \overline{q_{k}}$ and $\overline{q_{l}}$, we would have a $\mathbf{1 0}$ and $\overline{\mathbf{1 0}}$ of mesons with a $P$-wave internally. The $\pi_{1}(1400 ; 1600)$ could be members of such a multiplet (this was originally proposed on symmetry grounds in ref [13]); however, an inescapable consequence of such a proposal is that there exists an exotic $u d \bar{s} \bar{s}$ meson with strangeness +2 . With $m(\bar{s}) \sim m[u d]$ we predict this to be at $\sim 1600 \mathrm{MeV}$. Assuming that the $\Gamma\left(\Theta^{+}(1540)\right)$ is narrow due to a mixing between $\left([u d]_{1}^{6_{c}} \bar{s}\right)$ and $[u d]_{0}^{3_{c}} \bar{s}$ such that the low mass eigenstate decouples from $K N$, then the $\vartheta^{+}$should exist with a "normal" width. With a mass even at $1700 \mathrm{MeV}$ the phase space ratio for $\vartheta \rightarrow K \bar{K}$ and $\Theta^{+} \rightarrow K N$ is $\sim 16$. The $K K^{*}$ channel is open; the phase space enhancement in this case is $\sim 5$ but the spin counting will elevate this so that we may expect a similar branching ratio to that of the $K K$ mode. The three body $K K \pi$ mode will also contribute but uncorrelated three body modes are not expected to dominate over the two body ones. The net result is that we expect $\Gamma\left(\vartheta^{+}\right) \lesssim 100 \mathrm{MeV}$, such that the $\vartheta$ should be detectable (likewise for the equivalent $S=-2$ member of the 10).

Surprisingly it is not immediately possible to exclude such an exotic state in $K^{+} K^{0}$ if its mass is $\sim 1.6-1.7 \mathrm{GeV}$, and a dedicated search is suggested in e.g. $K^{+} N \rightarrow K^{+} K^{0} \Sigma[19]$. Other exotic members of the multiplets are probably broad and unobservable if the dynamics of ref [8] underpins the formation of triquarks (see later).

\footnotetext{
${ }^{5}$ Note that this is more reasonable than the $207 \mathrm{MeV}$ claimed by KL [4] on the basis of an analogy to the $D_{s}$ spectrum, see [17] for a critique.
} 


\begin{tabular}{l|c|c} 
& $\overline{\mathbf{1 0}}$ & $\mathbf{8}_{5}$ \\
\hline$\Theta^{+}$ & $A A A$ & $-(A C A+C A A-2 A A C) / \sqrt{6}$ \\
\hline$p$ & $-(A C A+C A A+A A C) / \sqrt{3}$ & $(A B A+B A A-2 A A B) / \sqrt{6}$ \\
$n$ & $(A B A+B A A+A A B) / \sqrt{3}$ & $(C A C+A C C-2 C C A) / \sqrt{6}$ \\
\hline$\Sigma^{+}$ & $(C A C+A C C+C C A) / \sqrt{3}$ & $-(A B C-A C B+B A C-C A B) / 2$ \\
$\Sigma^{0}$ & $-(A B C+B A C+A C B+C A B+B C A+C B A) / \sqrt{6}$ & $-(A B C+B A C+A C B+C A B-2 B C A-2 C B A) / \sqrt{12}$ \\
$\Lambda^{0}$ & $(B A B+A B B+B B A) / \sqrt{3}$ & $(B A B+A B B-2 B B A) / \sqrt{6}$ \\
$\Sigma^{-}$ & $-C C C$ & $(C B C+B C C-2 C B B) / \sqrt{6}$ \\
\hline$\Xi^{+}$ & $(C B C+B C C+C C B) / \sqrt{3}$ & $-(C B B+B C B-2 B B C) / \sqrt{6}$ \\
$\Xi^{0}$ & $-(C B B+B C B+B B C) / \sqrt{3}$ & \\
$\Xi^{-}$ & $B B B$ &
\end{tabular}

Table 1: Pentaquark wavefunctions where $A B C$ are defined in the text. Note that consistency requires the meson octet to be defined with each $q \bar{q}$ positive except for $\pi^{+}=-u \bar{d} ; \bar{K}^{0}=-s \bar{d}$ and then $\pi^{0}=(u \bar{u}-d \bar{d}) / \sqrt{2}$. In this convention $\eta_{8}=(2 s \bar{s}-u \bar{u}-d \bar{d}) / \sqrt{6}$

\section{Other members of the meson $10 \oplus \overline{\mathbf{1 0}}$}

A unified convention for constructing the symmetry states for multiquarks is given in Table 1 of [20], reproduced here as Table 1. This gives the combinations of three labels for the symmetric and mixed $(\lambda)$ states; the mixed $(\rho)$ and antisymmetric follow trivially. The labels $A, B, C$ are defined $A \equiv[u d]=\bar{s} ; B \equiv$ $[d s]=\bar{u} ; C \equiv[s u]=\bar{d}$; note that $[u d] \equiv(u d-d u) / \sqrt{2}$, etc., the sign of the antisymmetric combination being important.

In the meson case it is useful to adopt the order $A_{1} B_{2} \equiv([q q] \bar{q}), C_{3} \equiv \bar{q}$ and the (..) shows which are understood to be in the triquark correlation.

Triquarks $[q q] \bar{q}$ are in flavour $\overline{\mathbf{3}} \otimes \overline{\mathbf{3}}=\overline{\mathbf{6}} \oplus \mathbf{3}$. To make a $\overline{\mathbf{1 0}} \oplus \mathbf{8}$ of mesons (or pentaquarks) the triquark must be in flavour $\overline{\mathbf{6}}$, which is composed of the following members:

$$
\begin{gathered}
A A=[u d] \bar{s} \\
\{A B\}=([u d] \bar{u}+[d s] \bar{s}) / \sqrt{2} \quad\{C A\}=([u d] \bar{d}+[s u] \bar{s}) / \sqrt{2} \\
B B=[d s] \bar{u} \quad\{B C\}=([d s] \bar{d}+[s u] \bar{u}) / \sqrt{2} \quad C C=[s u] \bar{d}
\end{gathered}
$$

The nonexotic combinations in which $q_{i} q_{j} \overline{q_{j}}$ are in flavour 3 allow the possibility of $q_{j} \overline{q_{j}} \rightarrow$ gluons and hence mixing with conventional hadrons; such combinations have no advantages in forming metastable states and comprise the $P$-wave excited version of Jaffe's nonet [1]. The combinations $\{A B\},\{B C\}$ and $\{C A\}$ are at least stable against $q_{j} \overline{q_{j}} \rightarrow$ gluons (by $\left.S U(3)_{F}\right)$ although in the mechanism of Vento [8], it is only the $A A$ triquark that is fully stable, all others being unstable against decay into $\pi$ or $\eta_{s}$.

Using the conventions of [20], a full set of meson representations can be con- 
structed:

$$
\begin{array}{ll}
\vartheta^{+}=A A A & \\
K_{10}^{+}=-(A C A+C A A+A A C) / \sqrt{3} & K_{\mathbf{8 b}}^{+}=-(A C A+C A A-2 A A C) / \sqrt{6} \\
\pi_{\frac{10}{10}}^{+}=-(C A C+A C C+C C A) / \sqrt{3} & \pi_{\mathbf{8 b}}^{+}=-(C A C+A C C-2 C C A) / \sqrt{6} \\
" K^{+} "=C C C &
\end{array}
$$

The other charge combinations follow by acting on these with $I_{-}$accordingly. Charge conjugate analogues of these correspond to a $\mathbf{1 0} \oplus \mathbf{8}$.

Ref. [4] considered only the exotic states at the corner of the $\overline{\mathbf{1 0}}$. The dynamics of ref. [8], discussed earlier, suggest that other configurations, such as $([s u] \bar{d})$ (and $([d s] \bar{u}))$ are energetically unfavoured, due to the presence of $[u s]$ in place of $[u d]$, for which the instanton forces are less attractive, and unstable because $m([u s] \bar{d}) \geq m(\pi)+m(s)$, which enables decay. One consequence could appear to be that only the $\Theta^{+}$will be narrow in such a dynamics: the $\Xi^{+,--}$contain $([s u] \bar{d})$ or $([d s] \bar{u})$ which are unstable against decay into $\pi+s$, while the other states mix with 8 .

The absence of prominent signals other than the $\Theta^{+}$in the $\overline{\mathbf{1 0}}$, in particular the $\Sigma_{5}$ [21], may thus be explained: if such correlations occur, then they only create metastable configurations if either $[u d]$ and/or $([u d] \bar{s})$ are involved. We argue that the same situation arises in the $\overline{\mathbf{1 0}}$ and $\mathbf{1 0}$ of mesons: the only exotic combinations with a chance of suppressed widths are the $\vartheta^{+}$and $\vartheta^{-}$, containing the triquarks $A A$ and $\bar{A} \bar{A}$ respectively.

The exotic $S=-1 K^{+}([s u] \bar{d}) \bar{d}$ and $K^{--}([d s] \bar{u}) \bar{u}$ contain triquarks that are unstable against $\pi$ emission in the model of ref [8]. Thus we expect their widths to be at least $300 \mathrm{MeV}$. Identifying such states will be a challenge. The mass gap between the $\vartheta^{+}$and the $K^{+, 0,-,--}$ states will be $\sim 2 m(s-d)-m([u s]-[u d])$. As the $m([u s]-[u d])$ is likely to be at least as large as $m(s-d)$ the spread is likely to be only $\sim 100 \mathrm{MeV}$.

The other combinations occur in nonexotic multiplets and in general will mix. Consider for example the $J^{P}=1^{-}$states, $\rho^{0}$ or $\pi_{1}^{0}$. Depending on the mixing angle between the $\overline{\mathbf{1 0}}$ and $\mathbf{8}$ basis states, the mass eigenstates can be degenerate or separated by up to $2(m(s)-m(d))$. In this latter case the physical states are the ideal mass eigenstates $[q q] \bar{q} \bar{q}$ and $[s q] \bar{q} \bar{s}$. This small mass range for the states suggests that the hidden flavour mass basis is more representative than an $S U(3)_{F}$ multiplet basis.

Thus we need to count the number of states. For the neutral states with $I=1$ and $I=0$ there are six permutations of the distinct $A B C$ labels. In the $S U(3)_{F}$ symmetry basis these correspond to

$$
\begin{array}{ll}
\overline{\mathbf{1 0}}: I=1 ; & \mathbf{8}^{\lambda}:(I=1)+(I=0) ; \\
\mathbf{8}^{\rho}:(I=1)+(I=0) ; & \mathbf{1}: I=0 .
\end{array}
$$

The latter trio correspond to the familiar $\rho ; \omega_{8} ; \omega_{1}$ combinations in the case of $1^{-}$and as such are indistinguishable from radially excited $q \bar{q}$ nonets. The former would correspond to a pair of $\rho$ s and a single $\omega_{8}$, and hence would be novel. Thus counting the population of $I=1$ and $I=0$ vector mesons within an 
energy range of $\sim 300 \mathrm{MeV}$ can hint at which underlying multiplets are present. While the $\rho(1700), \omega(1650), \phi(1680)$ form a candidate nonet, their masses are somewhat unnatural. The $\rho(1450)$ and $\omega(1420)$ are missing a partner to complete the set. Depending on whether this is $I=0$ or $I=1$ could be novel. The $K^{*}(1410)$ appears to be anomalously low in mass for $q \bar{q}$ systems but fits naturally into the $\overline{\mathbf{1 0}}$ configuration. The widths of most of these states are hundreds of $\mathrm{MeV}$.

The general feature is that for a given $J^{P(C)}$ six neutral members are expected within a few hundred $\mathrm{MeV}$. If any of the plethora of observed states [9] is associated with these, such that their widths of $\sim 300 \mathrm{MeV}$ give a scale for their (in)stability, then a $\vartheta^{+}$seems an unavoidable consequence.

\section{Dynamics and $J^{P C}$ in $L=1 q q \bar{q} \bar{q}$ mesons}

An $L=1 q q \bar{q} \bar{q}$ system has a variety of $J^{P C}$ combinations. The relative masses and potential stability of these can depend upon the correlations of strong QCD. We now investigate the different dynamical arrangements for an $L=1 q q \bar{q} \bar{q}$ system, distinguished by the configuaration of the orbital angular momentum. We will see that dynamics may favour a triquark configuration for the $\mathbf{1 8} \oplus \overline{\mathbf{1 8}}$ of mesons, and that such a configuration has a limited spectum of $J^{P C}$ states. The same dynamical assumptions, namely the prevalence of "good" diquarks over "bad", explains the absence of higher representations such as $\mathbf{2 7}$.

In the diquark-diquark correlation, the $q q$ and $\bar{q} \bar{q}$ systems are each in $L=0$ with an $L=1$ between them. For quark pairs which are symmetric in space, the remaining degrees of freedom must be antisymmetric. The allowed combinations are as follows, for total quark spin $S$, with $\{\quad\}$ and [ ] denoting flavour $6(\overline{\mathbf{6}})$ and $\mathbf{3}(\overline{\mathbf{3}})$ respectively, and superscripts and subscripts denoting colour and spin:

$$
\mathbf{1 0}+\mathbf{8} \quad \overline{\mathbf{1 0}}+\mathbf{8}
$$

(i) $\quad\{q q\}_{1}^{\overline{3}}[\bar{q} \bar{q}]_{0}^{3} \quad[q q]_{0}^{\overline{3}}\{\bar{q} \bar{q}\}_{1}^{3} \quad S=1, J^{P}=0^{-}, 1^{-}, 2^{-}$

(ii) $\{q q\}_{0}^{6}[\bar{q} \bar{q}]_{1}^{6} \quad[q q]_{1}^{6}\{\bar{q} \bar{q}\}_{0}^{6} \quad S=1, J^{P}=0^{-}, 1^{-}, 2^{-}$

A different set of configurations arises if the $L=1$ is between a pair of quarks or antiquarks and the concept of a "diquark" dissolves. The quark pairs are now spatially antisymmetric, so that to satisfy the Pauli principle, the same flavour and colour correlations as above will have spin symmetry flipped from 0 (antisymmetric) to 1 (symmetric), or vice versa. The resulting combinations, where $q \mid q$ denotes a pair of quarks in $L=1$, are: 


$\begin{array}{cccl}\mathbf{1 0}+\mathbf{8} & \overline{\mathbf{1 0}}+\mathbf{8} & \\ \text { (iii) } \quad\{q \mid q\}_{0}^{\overline{3}}[\bar{q} \bar{q}]_{0}^{3} & {[q q]_{0}^{3}\{\bar{q} \mid \bar{q}\}_{0}^{3}} & & S=0, J^{P}=1^{-} \\ \text {(iv) } & \{q \mid q\}_{1}^{6}[\bar{q} \bar{q}]_{1}^{6} & {[q q]_{1}^{6}\{\bar{q} \mid \bar{q}\}_{1}^{6}} & S=0, J^{P}=1^{-} \\ & & & S=1, J^{P}=0^{-}, 1^{-}, 2^{-} \\ & & S=2, J^{P}=0^{-}, 1^{-}, 2^{-}, 3^{-} \\ \text {(v) } \quad\{q q\}_{0}^{6}[\bar{q} \mid \bar{q}]_{0}^{\overline{6}} & {[q \mid q]_{1}^{\overline{3}}\{\bar{q} \bar{q}\}_{1}^{3}} & S=0, J^{P}=1^{-} \\ \text {(vi) } & \{q q\}_{1}^{3}[\bar{q} \mid \bar{q}]_{1}^{3} & {[q \mid q]_{0}^{6}\{\bar{q} \bar{q}\}_{0}^{6}} & S=0, J^{P}=1^{-} \\ & & & S=1, J^{P}=0^{-}, 1^{-}, 2^{-} \\ & & & S=2, J^{P}=0^{-}, 1^{-}, 2^{-}, 3^{-}\end{array}$

The first thing to notice is that the two different pictures have different $J$ couplings. If the diquark-diquark picture describes the $\pi_{1}(1400 ; 1600)$ mesons, then we would also expect $0^{-+}$and $2^{-+}$partners at comparable mass, shifted by spin orbit splittings. Conversely, the latter picture allows for $S=0,1$ or 2 . If we supposed that the dynamics were such that the $S=0$ state was favoured (and we will argue that this could be so), then the apparent absence of $0^{-+}$and $2^{-+}$ siblings to the $\pi_{1}$ mesons is natural.

In the diquark-diquark picture we see that a meson in $\mathbf{1 8}$ or $\overline{\mathbf{1 8}}$ is made of a "good" and "bad" diquark: in Jaffe's original paper [1], the absence of $S$-wave mesons in this flavour representation was due to these repulsive colourmagnetic interactions. By contrast, we see that in the second picture, in which the $L=1$ is "within" a diquark, the spatial antisymmetry annuls these repulsive forces, turning a "bad" diquark of a given flavour (colour-spin symmetric) into a "good" diquark (colour-spin symmetric), as in configurations (iii) and (iv) (or conversely turns a "good" diquark into a "bad" diquark in configurations (v) and (vi)). However, once the orbital angular momentum separates quarks, the short-range hyperfine interaction is heavily suppressed, so it is better to describe these $L=1$ diquarks as neither "good", nor "bad", but "null".

What then can we say about the dynamics of a $[q q]\{\bar{q} \mid \bar{q}\}$ system? On the one hand, we could consider this sytem a direct mirror image of the JW correlation [3] for the $\Theta^{+},(u d)|(u d) \quad \bar{s} \rightarrow \bar{q}| \bar{q} \quad[q q]$. However, the $[q q]_{0}^{3} \bar{q}$ and $[q q]_{1}^{6} \bar{q}$ systems in $S=1 / 2$ are very light due to attractive colourmagnetic and instanton forces [7] [8], so it is natural to consider the configurations (iii) and (iv) as a tightly bound $S$-wave $q q \bar{q}$ triquark in a relative $P$-wave with a $\bar{q}$, (along with their charge conjugates) ${ }^{6}$. Each of these systems has a "good" diquark, and the presence of a $\bar{q}$ in $S$-wave with these diquarks lowers the energy further.

Refs [7] and [6] noted that the colourmagnetic interaction mixes the $S=1 / 2$ $[q q]_{0}^{\bar{a}} \bar{q}$ and $[q q]_{1}^{6} \bar{q}$ triquarks (configurations (iii) and (iv)) to give the lowest eigenvalue $-21.88 C$ in the case of full $S U(3)_{F}$ symmetry, a considerably stronger attraction than the diquark-diquark system, for which the lightest configuration has an energy shift of $-16 / 3 C$. Ref. [8] noted that instanton forces cause the same mixing and lower the energy of the triquark correlation further.

\footnotetext{
${ }^{6}$ Provided the triquark is compact spatially, the difference between a $P$-wave between the antiquarks and a $P$-wave between the free antiquark and the triquark can be neglected
} 
Note also that the mixing of the (iii) and (iv) configurations, with their downward energy shifts, occur only in the $S=0, J^{P}=1^{-}$state. Thus if this correlation is dominant, the lowest lying multiplets can be those with $J^{P}=1^{-}$, all other $J^{P}$ states being higher in energy. In any event, this possible dynamical picture limits the spectrum of $J^{P C}$ that could be expected, so that this picture is less readily falsified by the dearth of experimental evidence of mesons in $\mathbf{1 0}$ or $\overline{\mathbf{1 0}}$.

The same dynamics suggests that higher representations such as $\mathbf{6} \otimes \mathbf{6}=\mathbf{2 7} \oplus \mathbf{8} \oplus \mathbf{1}$ are not dynamically favoured. $\{q q\}\{\bar{q} \bar{q}\}$ systems are made of pairs of "bad" diquarks, and even moving to the triquark picture cannot annul these repulsive forces.

Thus we suggest that although tetraquark states in general will tend to be broad, the $J^{P}=1^{-} \overline{\mathbf{1 0}}$ and $\mathbf{1 0}$, possibly mixed with $\mathbf{8}$, may have the best chance of being observable. There are subtleties involved with forming the $C= \pm$ eigenstates associated with $\mathbf{1 8} \oplus \overline{\mathbf{1 8}}$; these are discussed in an appendix. We shall see later that the multiquark dynamics leading to the above also imply that there exists a "mirror" multiplet with $J^{P C}=1^{--}$neutral members.

The phenomonology of the controversial $\pi_{1}(1400)$ and $\pi_{1}(1600)$ is not inconsistent with the triquark correlation. The dynamically preferred arrangement in triquark language has $S=0$, which fits in neatly with the absence of clear $0^{-}$ and $2^{-}$partners to the $\pi_{1}(1400)$ and $\pi_{1}(1600)$. It is plausible that the mixed $[q q]_{0}^{3} \bar{q}$ and $[q q]_{1}^{6} \bar{q}$ system could be the only stable triquark correlation, and that states which do not benefit from this mixing $(S=1,2)$ are not dynamically preferred.

\section{$J^{P C}=1^{- \pm}$multiplets in correlated quark models}

In the search for evidence of gluonic degrees of freedom in Strong QCD, attention has focussed in particular on the prediction of exotic quantum numbers such as $J^{P C}=1^{-+}$, which are forbidden for $q \bar{q}$ in a potential but allowed if the gluonic degrees of freedom are excited. The masses of the lightest such hybrids are predicted from lattice QCD to be above $1.8 \mathrm{GeV}$ [22]. Thus the appearance of $\pi_{1}(1600) \rightarrow \pi \eta^{\prime}$ is intriguing as this was long ago suggested as a selection rule by Lipkin (see citation to unpublished remarks by Lipkin in [23]) and then in a modern context in [24] where the decay of a hybrid $1^{-+}$was predicted to have $\pi \eta^{\prime}>\pi \eta$. A problem is that such a state could also occur from $q q \bar{q} \bar{q}$ and the mass pattern of the other members of the nonet would need to be identified in order to distinguish this from a canonical $q \bar{q} g$ hybrid. In this context there is also reported a companion state $\pi_{1}(1400)$, which is seen in $\pi \eta$ but not $\pi \eta^{\prime}$. If such a decay conserves flavour, and if $\eta \equiv \eta_{\mathbf{8}}$, then such a state cannot belong to an 8 . Motivated by this state, ref [13] proposed that $\pi_{1}(1400)$ belong to a $\mathbf{1 0} \oplus \overline{\mathbf{1 0}} q q \bar{q} \bar{q}$ multiplet, and thus is not a hybrid $q \bar{q} g$ state in $\mathbf{8}$.

As shown in the appendix, we can take a linear combinations of $\pi_{\mathbf{1 0}}^{+}$and $\pi_{\mathbf{1 0}}^{+}$ to give a $I^{G}\left(J^{P C}\right)=1^{-}\left(1^{-+}\right)$state overlapping to $\pi^{+} \eta_{\mathbf{8}}$, and likewise a com- 
binations of $\pi_{\mathbf{8}_{\mathbf{a}}}^{+}$and $\pi_{\mathbf{8}_{\mathrm{b}}}^{+}$overlapping to $\pi^{+} \eta_{\mathbf{1}}$. The combinations $C A C$ and $C C A$ (and charge conjugates) have 2 strange masses, while $A C C$ has 0 strange masses, so we can express the $S U(3)_{F}$ symmetry basis states $X$ and $Y$ for the $1^{-}\left(1^{-+}\right)$states as:

$$
\begin{aligned}
& X=\left(\pi_{\mathbf{1 0}}^{+} \oplus \pi_{\overline{\mathbf{1 0}}}^{+}\right) / \sqrt{2}=\left[\pi^{0} \eta_{\mathbf{8}}\right]=(\sqrt{2} q s \bar{q} \bar{s}+q q \bar{q} \bar{q}) / \sqrt{3} \\
& Y=\left(\pi_{\mathbf{8}_{\mathbf{a}}^{+}}^{+} \oplus \pi_{\mathbf{8}_{\mathbf{b}}}^{+}\right) / \sqrt{2}=\left[\pi^{0} \eta_{\mathbf{1}}\right]=(-\sqrt{2} q s \bar{q} \bar{s}+2 q q \bar{q} \bar{q}) / \sqrt{6}
\end{aligned}
$$

where $q s \bar{q} \bar{s}$ and $q q \bar{q} \bar{q}$ denote the (normalised) parts of the wavefunction with 2 and 0 strange quarks respectively. The orthogonal linear combinations give an $I^{G}\left(J^{P C}\right)=1^{+}\left(1^{--}\right) \rho$ state overlapping to meson states $K \bar{K}$ and $\pi \pi$. For ref. [13], who assume $\eta \equiv \eta_{\mathbf{8}}$ and $\eta^{\prime} \equiv \eta_{\mathbf{1}}$, it is states $X, Y$ respectively that decay to $\pi \eta$ and $\pi \eta^{\prime}$ : the physical $\eta$ and $\eta^{\prime}$ are not pure $\mathbf{8}$ and $\mathbf{1}$. One could choose the mixing of the decuplet and octet states $X$ and $Y$ to enforce decays to the $\pi \eta$ and $\pi \eta^{\prime}$ respectively; this would require the mixing angle of $X, Y$ to be the same as the $\eta-\eta^{\prime}$ mixing. The eigenstates mixed in this way would then correspond to $\pi_{1}(1400) \rightarrow \eta \pi$ and $\pi_{1}(1600) \rightarrow \eta^{\prime} \pi$. Conversely, mass eigenstates that are ideal

$$
\begin{aligned}
X_{H} & =q q \bar{q} \bar{q} \\
X_{L} & =q s \bar{q} \bar{s}
\end{aligned}
$$

will decay to $\pi \eta_{n}$ and $\pi \eta_{s}$ respectively.

In order to go from mass eigenstates to symmetry basis states, it is necessary to have a mixing amplitude $A[(d \bar{d} u) \rightarrow(ß u)]>m(d \bar{d} u)-m(ß u)$. The stability of the $B u$ in contrast to the $d \bar{d} u$ seems to argue against that but a definite answer is beyond our ability to determine without further assumptions. If $\pi_{1}(1400 ; 1600)$ are identified with these states, then the quark mass eigenstates appear to be nearly realised: the masses based on the simplest flavour counting are consistent with $\pi_{1}^{+}(1400)$ and $\pi_{1}^{+}(1600)$ as the (dominantly) $q q \bar{q} \bar{q}$ and $q s \bar{q} \bar{s}$ states. This agrees with our earlier prediction that $m(\vartheta)$, the $u d \bar{s} \bar{s}$ state is also $\sim 1600 \mathrm{MeV}$, which in turn is consistent with the $\Theta^{+}(1540)$. A pair of $\rho$ states with similar masses to those of the two $\pi_{1} \mathrm{~s}$ are required. There are known problems with identifying the $\rho(1460 ; 1600)$ as simply $q \bar{q}$ states $[25]$ and the existence of $\rho(1250)$ remains uncertain. If the latter exists as a $q \bar{q}$ candidate, then the other pair may be related to the $q q \bar{q} \bar{q}$ and $q s \bar{q} \bar{s}$ states. Conversely, if the $\rho(1460)$ is the lightest such resonance, then the $K^{*}(1410)$ mass is more in tune with the pattern of interest here than it would be for a $q \bar{q}$ nonet. A test will be the presence or absence of isoscalar partners to these states. A $\mathbf{1 0}$ or $\overline{\mathbf{1 0}}$ have no such $I=0$ $\eta_{1}$ or $\omega$ states whereas the $\mathbf{8}$ does; a canonical nonet would have the $\rho ; \omega ; \phi$ analogues.

In any case, the $\sim 300 \mathrm{MeV}$ width of the $\pi_{1}(1400)$ and $\pi_{1}(1600)$ is consistent with a triquark dynamics. The wavefunction of a $\pi$ state, in any decuplet-octet mixing scenario, is composed of triquarks that can decay to $\pi+s, \pi+q$ or $\eta_{s}+q$ (or charge conjugates, for the antitriquark component). 
In [13] it is shown that if the $1^{-+} \pi_{1}(1400) \rightarrow \eta \pi$ is a member of a $\overline{\mathbf{1 0}} \oplus \mathbf{1 0}$ then there must also be a partner state $\rho \rightarrow \pi \pi$ and $K \bar{K}$. Using symmetry arguments, ref. [13] show that this "supermultiplet" of $\mathbf{1 0} \oplus \overline{\mathbf{1 0}}$ decaying to two pseudoscalar mesons (PP) ought to be accompanied by another supermultiplet decaying to a pseudoscalar and vector meson $(\mathrm{PV})$, a $I^{G}\left(J^{P C}\right)=1^{+}\left(1^{--}\right) \rightarrow \omega \pi$ and a $1^{-}\left(1^{-+}\right) \rightarrow \rho \pi, K^{*} \bar{K}$. Note, however, that the $1^{- \pm}$in the PV supermultiplet mentioned by CKK in ref. [13] must be accompanied by siblings $0^{- \pm}$and $2^{- \pm}$. By visualising the system as a triquark-antiquark in a $P$-wave, we can immediately understand the origin of the two supermultiplets and their $J^{P C}$ quantum numbers.

In normal $q \bar{q}$ mesons, $q$ and $\bar{q}$ have opposite parity, so that $P$-wave states have $P=+$. Conversely, in the triquark-antiquark picture, the triquark $Q$ and antiquark $\bar{q}$ have the same parity, so that $P$-wave states have $P=-$. In the appendix, we show that the wavefunction of a $q q \bar{q} \bar{q}$ meson has an extra degree of freedom compared to a $q \bar{q}$ meson, manifested in the freedom to take $\mathbf{1 0}+\overline{\mathbf{1 0}}$ versus $\mathbf{1 0}-\overline{\mathbf{1 0}}$ (or equivalently $\mathbf{8}_{\mathrm{a}}+\mathbf{8}_{\mathrm{b}}$ versus $\mathbf{8}_{\mathrm{a}}-\mathbf{8}_{\mathrm{b}}$ ), giving $C=+$ and $C=-$ for each $J$. So if we take the $q \bar{q}$ spectrum of states,

$$
S=0, J=1^{+-} \quad S=1, J=0^{++}, 1^{++}, 2^{++}
$$

flip the parity, and take $C= \pm$, we acquire precisely the spectrum of [13]:

$$
\begin{aligned}
& S=0, J=1^{--} \quad S=1, J=0^{--}, 1^{--}, 2^{--} \\
& S=0, J=1^{-+} \quad S=1, J=0^{-+}, 1^{-+}, 2^{-+}
\end{aligned}
$$

In the work of Chung et al [13] there is no dynamical picture that distinguishes the two supermultiplets, and hence no suggestion as to why the PV supermultiplet has not been experimentally observed. We show in the appendix that there are different dynamics underpinning the supermultiplets. The PP supermultiplet, to which the observed $\pi_{1} \rightarrow \eta \pi$ belongs, has $S=0$, and we saw earlier that this spin configuration is the only one in which mixing from one gluon exchange and instanton forces allows a light and metastable triquark. On the contrary, the PV multiplet has $S=1$, a configuration in which mixing cannot occur, resulting in heavier, possibly unstable triquarks. This might account for the experimental elusiveness of the PV supermultiplet.

Earlier we noted that the diquark-diquark picture can only have $S=1, J=$ $0^{-}, 1^{-}, 2^{-}$. In the appendix we show that for the diquark-diquark configuration $\zeta=(-1)^{l}$, so that such a system in $P$-wave can exist in only one supermultiplet $(\zeta=-1)$. Due to angular momentum conservation in the decays of the $0^{-}$ and $2^{-}$, this supermultiplet can only be PV. Thus, provided it is valid to treat diquarks as effective bosons, the $1^{-+} \pi_{1}(1400) \rightarrow \eta \pi$ and $\pi_{1}(1600) \rightarrow \eta^{\prime} \pi$ cannot be in the diquark-diquark arrangement.

\section{Conclusion}

In conclusion, confirmation of a narrow $\Theta^{+}(1540)$ and the absence of other narrow members of a $\overline{\mathbf{1 0}}$ can be explained by correlations that suggest there 
should be a $\vartheta$ with canonical width $\lesssim 100 \mathrm{MeV}$ together with a family of broad partners. This particular dynamics is exceptional as received wisdom has been that all these states should "fall-apart". If the $\Theta^{+}$is an artefact, or if its narrow width is due to some mechanism other than the mixing among correlations as discussed here, then the $1^{-( \pm)}$tetraquark mesons will be all broad as in $[5,10]$. In any event, if the existence of a resonant $\Theta^{+}$with narrow width survives further scrutiny, then a $\overline{\mathbf{1 0}}$ of mesons with moderate widths, of which the $\vartheta$ may have a canonical width, merits investigation.

Models which consider four-quark mesons produce a considerable multiplicity of flavour and spin states. Using a triquark-quark correlation for tetraquarks there can be a exception to this rule, with only a reduced set of states appearing and the possibility that among these the $J^{P}=1^{-} \overline{\mathbf{1 0}}$ and $\mathbf{1 0}$ may contain observable states. It seems possible to associate certain otherwise peculiar states in the meson spectrum with those predicted here.

This model is trivially falsifiable by comparision of its prediction of flavour exotic states with experiment. We have noted that while the $\rho(1700), \omega(1650), \phi(1680)$ form a candidate nonet, their masses are somewhat unnatural. The $\rho(1450)$ and $\omega(1420)$ are missing a partner to complete the set and determine whether they are in a nonet or $\overline{\mathbf{1 0}}$. The $K^{*}(1410)$ appears to be anomalously low in mass for $q \bar{q}$ systems but fits naturally into the $\overline{\mathbf{1 0}}$ configuration. The widths of most of these states are hundreds of $\mathrm{MeV}$.

The general feature is that for a given $J^{P(C)}$ six neutral members are expected within a few hundred $\mathrm{MeV}$. If any of the plethora of observed states [9] is associated with these, such that their widths of $\sim 300 \mathrm{MeV}$ give a scale for their (in)stability, then a $\vartheta^{+}$with a canonical width seems an unavoidable consequence.

\section{Appendix}

To obtain charge conjugation eigenstates for tetraquark mesons we need to write their wavefunctions in $q q \bar{q} \bar{q}$ and $\bar{q} \bar{q} q q$ form. We demonstrate the procedure for the nonstange neutral members of the $\mathbf{1 0}$ and $\overline{\mathbf{1 0}}$, noting that we can easily generalise to the $I_{3}= \pm 1$ members with the usual $G$ parity operator. Note also that the following analyses work for the $\mathbf{8}_{\mathbf{a}}$ and $\mathbf{8}_{\mathbf{b}}$.

From the wavefunctions given earlier, we find

$$
\begin{aligned}
& \pi_{\mathbf{1 0}}^{0}=\mathbf{6} \otimes \mathbf{3}=-(\{d s\}[\bar{d} \bar{s}]+\{s u\}[\bar{s} \bar{u}]+\{u d\}[\bar{u} \bar{d}]) / \sqrt{3} \\
& \widetilde{\pi}_{\mathbf{1 0}}^{0}=\mathbf{3} \otimes \mathbf{6}=-([\bar{d} \bar{s}]\{d s\}+[\bar{s} \bar{u}]\{s u\}+[\bar{u} \bar{d}]\{u d\}) / \sqrt{3} \\
& \pi_{\overline{\mathbf{1 0}}}^{0}=\overline{\mathbf{3}} \otimes \overline{\mathbf{6}}=-([d s]\{\bar{d} \bar{s}\}+[s u]\{\bar{s} \bar{u}\}+[u d]\{\bar{u} \bar{d}\}) / \sqrt{3} \\
& \widetilde{\pi}_{\overline{\mathbf{1 0}} \mathbf{0}}^{\mathbf{0}} \overline{\mathbf{6}} \otimes \overline{\mathbf{3}}=-(\{\bar{d} \bar{s}\}[d s]+\{\bar{s} \bar{u}\}[s u]+\{\bar{u} \bar{d}\}[u d]) / \sqrt{3}
\end{aligned}
$$

Notice $C \pi_{\mathbf{1 0}}^{0}=\widetilde{\pi}_{\overline{\mathbf{1 0}}}^{0}$ and $C \pi_{\overline{\mathbf{1 0}}}^{0}=\widetilde{\pi}_{\mathbf{1 0}}^{0}$, so if we denote the $\mathbf{1 0}$ and $\overline{\mathbf{1 0}}$ wavefunctions

$$
\chi(\mathbf{1 0}, \zeta)=\pi_{\mathbf{1 0}}^{0}+\zeta \widetilde{\pi}_{\mathbf{1 0}}^{0} \quad \chi(\overline{\mathbf{1 0}}, \zeta)=\pi_{\overline{\mathbf{1 0}}}^{0}+\zeta \widetilde{\pi}_{\overline{\mathbf{1 0}}}^{0}
$$


then our $\zeta$ is precisely that defined by equation (2) in [13]:

$$
C \chi(\mathbf{1 0}, \zeta)=\zeta \chi(\overline{\mathbf{1 0}}, \zeta) \quad C \chi(\overline{\mathbf{1 0}}, \zeta)=\zeta \chi(\mathbf{1 0}, \zeta)
$$

We have the freedom to choose the phase between the $\mathbf{1 0}$ and $\overline{\mathbf{1 0}}$, which we denote by $a$, so that the full wavefunctions of a four quark $\mathbf{1 0} \oplus \overline{\mathbf{1 0}}$ have two degrees of freedom, $a$ and $\zeta$,

$$
\left.\pi^{0}(\zeta, a)=\pi_{\mathbf{1 0}}^{0}+\zeta \widetilde{\pi}_{\mathbf{1 0}}^{0}+a\left(\pi_{\mathbf{1 0}}^{0}+\zeta \widetilde{\pi}_{\overline{\mathbf{1 0}}}^{0}\right)\right)
$$

We see that $C \pi^{0}(\zeta, a)=\zeta a \pi^{0}(\zeta, a)$ and the doubling of states follows: in the $\zeta=-1$ multiplet we have a $1^{--}(\zeta a=-+)$ and $1^{-+}(\zeta a=--)$, and in the $\zeta=+1$ multiplet we have a $1^{--}(\zeta a=+-)$ and $1^{-+}(\zeta a=++)$. Looking at the $q q \bar{q} \bar{q}$ part of the wavefunction only, we see that under interchange of quarks $2 \leftrightarrow 3$ linear combinations of $\pi_{10}^{0}$ and $\pi_{10}^{0}$ correspond to CKK's flavour wavefunctions in $q \bar{q} q \bar{q}$ form: the $1^{-+}$state is $\pi_{10}^{0}+\pi_{\overline{10}}^{0} \equiv\left[\pi^{0} \eta_{8}\right]$, the $1^{--}$state is $\pi_{\mathbf{1 0}}^{0}-\pi_{\overline{\mathbf{1 0}}}^{0} \equiv \sqrt{\frac{1}{3}}\left(\left[\pi^{-} \pi^{+}\right]+\left[\overline{K^{0}} K^{0}\right]+\left[K^{-} K^{+}\right]\right)$(our $\pi^{+}$is defined as $-u \bar{d}[20]$, hence the phase difference compared to CKK). Likewise for linear combinations of the wavefunctions $\mathbf{8}_{\mathbf{a}}$ and $\mathbf{8}_{\mathrm{b}}$.

We can think of a $P$-wave tetraquark meson as a system of two quasi particles in $L=1$ : in the diquark-diquark picture, two bosons of spin 0 and 1 , in the triquark picture, two fermions of spin $1 / 2$ (we assume the lightest triquark is spin $1 / 2$ following [13] [8]). In the JW picture of the $\Theta^{+}$[3], the $(u d)$ pairs are treated as effective bosons so that bose statistics forces a $P$-wave. However, this picture suffers from the fact that the uudd wavefunction is not fully antisymmetrised, only the $(u d)$ pairs individually. In the meson world there is no such problem. We can treat a $q q \bar{q} \bar{q}$ system as a pair of bosons or pair of fermions at the same time ensuring their fermionic wavefunctions are fully antisymmetrised.

Let us look firstly at the diquark-diquark correlation. Denoting $\{q q\}$ by $b$ and $[q q]$ by $B$, in shorthand notation we can write $\pi_{10}^{0}=b \bar{B}, \widetilde{\pi}_{10}^{0}=\bar{B} b, \pi_{10}^{0}=B \bar{b}$, $\widetilde{\pi}_{\overline{10}}^{0}=\bar{b} B$,so that the full wavefunctions can be expressed

$$
\pi^{0}(\zeta, a)=b_{1} \bar{B}_{2}+\zeta \overline{B_{1}} b_{2}+a\left(B_{1} \overline{b_{2}}+\zeta \overline{b_{1}} B_{2}\right)
$$

where the labels 1 and 2 denote the spin and space degrees of freedom of the bosons. $C \pi^{0}(\zeta, a)=\zeta a \pi^{0}(\zeta, a)$ in this shorthand notation, as expected. Interchanging the space and spin labels in the wavefunction brings a factor $(-1)^{l}$

$$
\pi^{0}(\zeta, a)=(-1)^{l}\left[b_{2} \bar{B}_{1}+\zeta \overline{B_{2}} b_{1}+a\left(B_{2} \overline{b_{1}}+\zeta \overline{b_{2}} B_{1}\right)\right]
$$

and since bosons are commuting variables $\left(\left[b_{1}, \bar{B}_{2}\right]=0\right)$,

$$
\begin{aligned}
\pi^{0}(\zeta, a) & =(-1)^{l}\left[\bar{B}_{1} b_{2}+\zeta b_{1} \overline{B_{2}}+a\left(\overline{b_{1}} B_{2}+\zeta B_{1} \overline{b_{2}}\right)\right] \\
& =\zeta(-1)^{l} \pi_{1}^{0}(\zeta, a)
\end{aligned}
$$

so in the diquark-diquark picture $\zeta=(-1)^{l}$. 
In the triquark-antiquark configuration there are two spin $1 / 2$ fermions in $L=1$. Denoting the triquarks $\{A B\},\{C A\}$ and $\{B C\}$ by $U, D$ and $S$,

$$
\begin{aligned}
& \pi_{10}^{0}=(-u \bar{U}+d \bar{D}+s \bar{S}) / \sqrt{3}=q \bar{Q} \\
& \widetilde{\pi}_{10}^{0}=(-\bar{U} u+\bar{D} d+\bar{S} s) / \sqrt{3}=\bar{Q} q \\
& \pi_{\overline{10}}^{0}=(-U \bar{u}+D \bar{d}+S \bar{s}) / \sqrt{3}=Q \bar{q} \\
& \widetilde{\pi}_{\overline{10}}^{0}=(-\bar{u} U+\bar{d} D+\bar{s} S) / \sqrt{3}=\bar{q} Q
\end{aligned}
$$

and the full wavefunction is

$$
\begin{aligned}
\pi^{0}(\zeta, a) & =q_{1} \bar{Q}_{2}+\zeta \overline{Q_{1}} q_{2}+a\left(\overline{Q_{1}} \overline{q_{2}}+\zeta \overline{q_{1}} Q_{2}\right) \\
& =(-1)^{s+1}(-1)^{l}\left[q_{2} \bar{Q}_{1}+\zeta \overline{Q_{2}} q_{1}+a\left(Q_{2} \overline{q_{1}}+\zeta \overline{q_{2}} Q_{1}\right)\right] \\
& =(-1)^{l+s}\left[\bar{Q}_{1} q_{2}+\zeta q_{1} \overline{Q_{2}}+a\left(\overline{q_{1}} Q_{2}+\zeta Q_{1} \overline{q_{2}}\right)\right] \\
& =(-1)^{l+s} \zeta \pi_{1}^{0}(\zeta, a)
\end{aligned}
$$

since interchanging the labels 1 and 2 brings factors $(-1)^{s+1}$ and $(-1)^{l}$ for the spin and space labels respectively, and for fermions $\left\{q_{1}, \bar{Q}_{2}\right\}=0$. Thus in the triquark antiquark picture $\zeta=(-1)^{l+s}$. Once again $C \pi^{0}(\zeta, a)=\zeta a \pi^{0}(\zeta, a)$ in this shorthand notation, as expected.

In the work of Chung et al [13], a quantum number $\zeta$ distinguishes the two supermultiplets, being labelled by $\zeta=+1(\mathrm{PP})$ and $\zeta=-1(\mathrm{PV})$, or vice versa (the overall phase of $\zeta$ is not important). At first glance, it appears as though the total spin $S$ of the quarks distinguishes the two supermultiplets: the $S=0$ states being $\mathrm{PP}$ and the $S=1$ states being PV, but some caution is needed. By angular momentum conservation for $P$-wave decays, $0^{-}, 2^{-} \rightarrow \mathrm{PV}$ but not to $\mathrm{PP}$, so we can immediately assign the $0^{-}$and $2^{-}$states to the PV multiplet. However, since we can have both $1^{-} \rightarrow \mathrm{PV}$ and $\rightarrow \mathrm{PP}$ in $\mathrm{P}$ wave, there is nothing, a priori, that tells us to which supermultiplet a $1^{-}$must belong. In the appendix, though, we use Fermi-Dirac statistics to show that for a $P$-wave triquark-antiquark system, the supermultiplet label $\zeta$ is given by $\zeta=(-1)^{s+1}$. We can confirm, then, what we had already suspected: that the total spin $S$ of the quarks determines precisely to which supermultiplet a state belongs. The states with $S=0$ belong to the PP supermultiplet $(\zeta=-1)$, and those with $S=1$ to the PV supermultiplet $(\zeta=+1)$.

As a brief digression, it's worth considering the $P$-wave excited version of Jaffe's original nonet in the triquark-antiquark correlation. In this case, we must have a flavour 3 of triquarks, where $S U(3)_{F}$ does not protect the annihilation of $q \bar{q}$ pairs, so stable triquarks cannot form. Annihilation of $q_{i} q \bar{q} \rightarrow q_{i}$ is equivalent to rewriting $U \rightarrow u, D \rightarrow d$ and $S \rightarrow s$, or simply $Q \rightarrow q$ in our shorthand notation. In that case, the wavefunction is clearly zero for $a=-1$. The extra degree of freedom has been stripped away, and we recover the ordinary spectrum of $q \bar{q}$ states:

$$
\begin{array}{ll}
\zeta a=-+ & S=0, J=1^{+-} \\
\zeta a=++ & S=1, J=0^{++}, 1^{++}, 2^{++}
\end{array}
$$


Thus in the triquark-antiquark picture, a nonet of $P=-$ tetraquarks will not form. On the contrary, in the diquark-diquark picture the $q \bar{q}$ annihilation is inhibited by the $P$-wave barrier so it is possible that a nonet should be seen.

\section{Acknowledgments}

This work is supported, in part, by grants from the Particle Physics and Astronomy Research Council, the EU-TMR program "Euridice" HPRN-CT-200200311, a Clarendon Fund Bursary and by the U.S. Department of Energy under contract DE-AC05-84ER40150.

\section{References}

[1] R.L.Jaffe, Phys.Rev. D 15267 (1977); F.E.Close and N.Tornqvist, J.Phys.G. 28 R249 (2002)

[2] A.De Rujula, H.Georgi and S.L.Glashow, Phys. Rev. D12, 147 (1975);Ya.B. Zeldovich and A.D. Sakharov, Yad. Fiz 4(1966)395; Sov. J. Nucl. Phys. 4(1967)283.

[3] R.L.Jaffe and F.Wilczek, hep-ph/0307341

[4] M.Karliner and H.J.Lipkin, hep-ph/0307243

[5] R.L. Jaffe, hep-ph/0409065

[6] B.Jennings and K.Maltman, hep-ph/0308286

[7] H.Hogaasen and P.Sorba, hep-ph/0406078

[8] N.I.Kochelev, H.J.Lee and V.Vento, hep-ph/0404065

[9] Particle Data Group, Phys Letters B592 (2004) 1

[10] R.L.Jaffe, Phys.Rev. D 15267 (1977)

[11] F.E.Close and N.Tornqvist, J.Phys.G. 28 R249 (2002)

[12] L.Maiani, F.Piccinini, A.D.Polosa, V.Riquer, hep-ph/0407017

[13] S.U.Chung, E.Klempt and J G Korner, hep-ph/0211100 S.U.Chung and E.Klempt, hep-ph/0306018

[14] A. R. Dzierba et al., Phys. Rev. D 67 (2003) 094015

[15] F.E.Close, Rapporteur talk at ICHEP04

[16] M.Karliner and H.J.Lipkin, Phys.Lett.B 586 303, 2004

[17] J.J.Dudek talk at the First Meeting of the APS Topical Group on Hadronic Physics, Fermilab November 2004 (Proceedings to appear in J.Phys.G) 
[18] F.E.Close and J.J.Dudek, Phys.Lett.B 583 278, 2004

[19] W.P.Dodd, T.Joldersma, R.B.Palmer and N.P.Samios, Phys. Rev. 177 (1969) 1991.

[20] F.E.Close and J J Dudek, hep-ph/0401192

[21] F.E.Close and Qiang Zhao, hep-ph/0404075

[22] P. Lacock, C. Michael, P. Boyle and P. Rowland, Phys.Lett. B 401, 308 (1997); C. Bernard et al., Phys.Rev. D 56, 7039 (1997); P. Lacock and K. Schilling, Nucl. Phys. Proc. Suppl. 73, 261 (1999); C. McNeile, hep-lat/9904013 C. Morningstar, Nucl. Phys. Proc. Suppl. 90, 214 (2000)

[23] B W Lee, S Okubo and J Schecter Phys Rev. 135 (1964) B219

[24] F.E.Close and H.J.Lipkin,Phys Lett 196 (1987) 245;

[25] F.E. Close, A. Donnachie and Yu.S. Kalashnikova, Phys.Rev. D 65, 092003 (2002) 